\title{
Estimativa da densidade básica da madeira de Eschweilera odora (Poepp.) Miers por espectroscopia no infravermelho próximo
}

\author{
Claudete Catanhede do Nascimento ${ }^{1 \star}$, Marta Martins Brasil ${ }^{1}$, Cristiano Souza do Nascimento ${ }^{1}$, Sâmia \\ Valéria dos Santos Barros ${ }^{2}$ \\ ${ }^{1}$ Laboratório de Engenharia e Artefatos de Madeira, Instituto Nacional de Pesquisas da Amazônia - INPA/COTI, Manaus, \\ Amazonas, Brasil. \\ ${ }^{2}$ Instituto Brasileiro do Meio Ambiente e dos Recursos Naturais Renováveis - IBAMA, Manaus, Amazonas, Brasil.
}

\begin{abstract}
RESUMO A densidade básica é uma das propriedades mais estudadas na tecnologia da madeira, visto sua importância na correlação com as propriedades físicas e mecânicas. A metodologia tradicional (destrutiva) demanda altos custos e extenso período para execução dos ensaios. Neste sentido, a pesquisa desenvolvida buscou determinar a densidade da madeira de Eschweilera odora por metodologia não destrutiva, avaliando a eficiência da espectroscopia no infravermelho próximo - NIR. Madeiras provenientes de floresta secundária de terra firme (Amazonas/Brasil) foram selecionadas para estudo, onde oito replicadas da espécie Eschweilera odora foram utilizadas para validação da densidade e 11 espécies (64 amostras) utilizadas na construção do modelo PLS/NIR. Os espectros NIR das madeiras foram coletados na faixa de 4.000-10.000 $\mathrm{cm}^{-1}$, onde a análise espectral possibilitou associar espectros $x$ densidade. Os modelos utilizados para estimar a densidade de Eschweilera odora nas faces tangenciais e radiais apresentaram altos índices de determinação $\left(\mathrm{R}^{2}>0,90\right)$ e baixos erros (Calibração/RMSEC e previsão/RMSEP). $\mathrm{O}$ teste $\mathrm{T}$ mostrou que a diferença entre os valores da densidade de Eschweilera odora preditos pelos PLS Radial e Tangencial não foi significativa para um intervalo de confiança de $95 \%$, apresentando resultados aproximados. Estes resultados confirmam a potencialidade da espectroscopia NIR para estimativa da densidade da madeira de Eschweilera odora.

Palavras-chave: madeiras da Amazônia; caracterização tecnológica; NIR; metodologia não destrutiva.
\end{abstract}

\section{Estimation of the basic density of wood Eschweilera odora (Poepp.) Miers by near infrared spectroscopy}

\begin{abstract}
The basic density is one of the most studied properties in wood technology area due to its significance in the correlation with the physical and the mechanical properties. The traditional (destructive) methodology demands high costs and an extended time period for the execution of the tests. The research developed here determines the basic density of Eschweilera odora wood by non-destructive methodology, evaluating the efficiency of the near infrared spectroscopy - NIR technique. Timber from "terra firme" secondary forest (Amazonas/Brazil) was selected for our study, where eight replicates of Eschweilera odora species were used for density's validation and 11 species (64 samples) were used to construct the PLS/NIR model. The NIR spectra of the woods were collected in the range of 4,000-10,000 $\mathrm{cm}^{-1}$, where the spectral analysis made it possible to associate spectra $\mathrm{x}$ density. The models used to determine the density of Eschweilera odora wood on the tangential and radial faces presented high determination indices $\left(\mathrm{R}^{2}>0.90\right)$ and low errors (Calibration/RMSEC and prediction/RMSEP). The T test showed that the difference between values of Eschweilera odora wood density predicted by Radial PLS and Tangential PLS were not significant $95 \%$ confidence level, showing approximate results. These results confirm the potential the NIR spectroscopy to estimate the wood density.
\end{abstract}

Keywords: Amazonian woods; technological characterization; NIR; Non-Destructive Methodology 


\section{Introdução}

A caracterização tecnológica de espécies madeireiras para fins de comercialização e confecção de produtos é realizada com base em normas nacionais e internacionais, tais como ABNT, COPANT e ASTM. Estas normativas prescrevem a necessidade de derrubar três a cinco árvores por espécie para obtenção de corpos de prova, com objetivo de determinar as propriedades físicas e mecânicas, por método convencional (STANGERLIN et al., 2015), não havendo possibilidade de uso posterior das amostras. A densidade básica é uma das propriedades mais estudadas na tecnologia da madeira, visto sua importância na correlação com as propriedades físicas e mecânicas, aliada a uso da madeira na construção civil, na indústria de celulose e papel e como fonte de combustível (NASCIMENTO, 2000; PIGOZZO, 2009; LIRA et al., 2010).

A metodologia tradicional (destrutiva) em geral demanda altos custos, extenso período para sua execução, que vai desde a etapa da coleta até a realização dos ensaios para caracterização. Neste sentido várias pesquisas estão sendo desenvolvidas em busca de novas metodologias para sanar tal problemática, surgindo em 2000 o termo avaliação não destrutiva - NDE. As pesquisas na área florestal resultaram no desenvolvimento de técnicas e equipamentos para predição de propriedades da madeira sem a necessidade de sua destruição (BODIG, 2000; ROSS et al., 2000; OLIVEIRA et al., 2002; BIEKER; RUST, 2010).

A aplicação dos métodos não destrutivo para madeiras possibilita uma melhor determinação de suas características tecnológicas, esta ferramenta vem auxiliar no conhecimento da variabilidade entre indivíduos e a caracterização de material no campo. Várias pesquisas têm sido desenvolvidas com o objetivo de predizer as propriedades da madeira por meio de métodos não destrutivo. Niemz; Mannes (2012) realizaram revisão sobre as principais NDE. Tanasoiu et al. (2002) avaliaram com sucesso as propriedades de estruturas de madeiras para construção utilizando método ultrassônico. Sanabria et al. (2011) utilizou também ondas ultrassônicas para avaliar defeitos em madeira laminada colada. Zauner et al. (2011) utilizando tomografia/sincroton conseguiu predizer a deformação de estruturas de madeiras quando expostas a testes mecânicos. Gouvêa et al. (2011), utilizou três técnicas NDE (Resistógrafo, Pilodyn e Espectroscopia de Infravermelho Próximo) para estimativa da densidade básica de madeiras de Eucalyptus sp., confirmando a aplicabilidade dessa ferramenta, inclusive peças em uso.

Dentre os métodos não destrutivo, os mais promissores para a indústria florestal são os espectroscópicos, que se baseiam nos ajustes de modelos preditivos e possuem como variáveis dependentes as propriedades da madeira e independentes a reflectância ou absorbância da radiação eletromagnética (SANTOS et al., 2009; HEIN et al., 2010; SOUSA et al., 2011).

A espectroscopia na região do infravermelho próximo (NIR) tem sido frequentemente aplicada como método analítico que fornece resultados eficientes para a determinação de moléculas orgânicas e variáveis quantitativas. A ferramenta NIR está presente em quase todas as áreas do conhecimento, devido ao nível de desenvolvimento de tecnologia, principalmente nos setores agrícola, alimentício, têxtil, cosméticos de polímeros, petroquímica, farmacêutica, ambiental e florestal (LEBLON et al., 2013; MUÑIZ et al., 2013; SANTOS et al., 2015).

A ferramenta NIR baseia-se na espectroscopia vibracional, com exposição de várias amostras a radiação eletromagnética na faixa de 4.000 a $12.000 \mathrm{~cm}^{-1}(750$ a $2.500 \mathrm{~nm})$, para obtenção dos espectros. Esta energia quando aplicada na madeira fornece informações que são associadas às várias propriedades físicas e mecânicas, possibilitando a combinação de variáveis desta matéria-prima (HAUKSSONET al., 2001; 
SCHIMLECK et al., 2001; TSUCHIKAWA et al., 2003; KEL-

LEY et al., 2004; TSUCHIKAWA, 2007), bem como dos seus constituintes químicos (KELLEY et al., 2004; TERDWONGWORAKUL et al., 2005; KRONGTAEW et al., 2010), e também anatômicos (JONES et al., 2005; TSUCHIKAWA, 2007; MUÑIZ et al., 2016), sendo que a maiorias das pesquisas são desenvolvidas com espécies de florestas plantadas e nativas de zona temperada.

Em relação ao uso da ferramenta NIR na caracterização de madeiras tropicais em especial da região amazônica, poucos são os registros nesta área (NASCIMENTO, et al., 2012a; SILVA et al., 2012; VAREJÃO et al., 2012; NASCIMENTO; BRASIL, 2016a). Modelo preditivo NIR para densidade básica da madeira de seis clones de Eucalyptus com três anos de idade de diferentes localidades apresentaram parâmetros quimiométricos satisfatórios, $\mathrm{R}^{2}=0,84$, baixos erros de calibração e predição $\left(0,013 \mathrm{~g} / \mathrm{cm}^{3}, 0,017 \mathrm{~g} / \mathrm{cm}^{3}\right.$, respectivamente). Neste mesmo estudo foi comparada a precisão do modelo NIR com o método Pilodyn, onde a espectroscopia apresentou maior precisão (GOUVÊA et al., 2011). Pigozzo (2009), desenvolveu calibrações com o espectro NIR para estimar a densidade básica de madeiras de diferentes espécies nativas e plantadas no Brasil, o tratamento matemático com a $2^{\mathrm{a}}$ derivada resultou num coeficiente de determinação $R^{2}=0,81$ e erro de predição $=0,062 \mathrm{~g} / \mathrm{cm}^{3}$ e de calibração $=0,058 \mathrm{~g} / \mathrm{cm}^{3}$. Neste contexto, visou-se avaliar a eficiência da espectroscopia no infravermelho próximo para predizer a densidade da madeira de Eschweilera odora.

\section{Material e Métodos}

A pesquisa foi realizada com madeiras provenientes 1 ha de área/floresta secundária de terra firme, localizada na Estação Experimental de Silvicultura Tropical de propriedade do INPA/BRASIL $\left(02^{\circ} 35^{\prime} 55,5^{\prime \prime} \mathrm{S}\right.$ e $\left.60^{\circ} 02^{\prime} 14,8^{\prime \prime} \mathrm{W}\right)$, no $\mathrm{km} 23$ da vicinal ZF-2, com acesso no km 50 na BR-174 (Manaus Boa Vista). Foram utilizadas 11 espécies florestais madeireiras (Tabela 1), e obtidos corpos de prova na secção transversal (2 x $2 \times 3 \mathrm{~cm}$ ), totalizando 64 amostras. As espécies utilizadas neste estudo estão sendo manejadas dentro do projeto INCT Madeiras da Amazônia - MCTIC/CNPq/FAPEAM.

A densidade $(\mathrm{Db})$ foi determinada conforme descrito na

Tabela 1. Espécies utilizadas no desenvolvimento dos modelos de predição NIR da densidade básica e na estimativa não destrutiva da Eschweilera odora.

Table 1. Species used in the development of the NIR prediction models of the basic density and in the non-destructive estimation of Eschweilera odora.

\begin{tabular}{lll}
\hline \multicolumn{1}{c}{ Nome comercial } & \multicolumn{1}{c}{ Nome científico } & Família \\
\hline Abiurana & $\begin{array}{l}\text { Manilkara amazoniaca (Huber.) Standley } \\
\text { Pouteria guianensis Aubl. }\end{array}$ & Sapotaceae \\
\hline Breu vermelho & $\begin{array}{l}\text { Protium tenuifolium Engl. } \\
\text { Protium puncticulatum Macbr }\end{array}$ & Burseraceae \\
\hline & Inga sp. & Mimosaceae \\
Ingá vermelha & Inga paraenses Ducke & \\
\hline Muiragiboia & Swartzia recurva Peopp Sw.) Willd. & Fabaceae \\
Murici & Byrsonima crispa Juss. & Malpighiaceae \\
Peãozinho & Micrandopsis scleroxylon W. Rodr. & Euphorbiaceae \\
Matá-matá & Eschweilera odora (Poepp.) Miers & Lecythidaceae \\
\hline
\end{tabular}


norma da Associação Brasileira de Normas Técnicas (ABNT) NBR 7190:1997. O método consiste no deslocamento da água (Figura 1). Os corpos de prova foram saturados para obter o volume verde, em seguida foram secos em estufa $\left(100^{\circ} \mathrm{C} \pm 3\right)$ até atingirem peso constante. Posteriormente, calculou-se a densidade básica conforme a formula: $\mathrm{Db}=\mathrm{Ps} / \mathrm{Vv}$, onde Ps equivale o peso seco em gramas, $\mathrm{Vv}$ o volume da amostra em estado saturado $\mathrm{em}^{\mathrm{cm}}$.

Os espectros na região do infravermelho próximo foram obtidos das amostras $(\mathrm{U}=12 \%)$, nos planos radial e tangencial, identificados faixa alburno/cerne, em espectrômetro com transformada de Fourier (FT-NIR/Termo Fisher Scientific, modelo Antaris II). O sistema utiliza o software Result Integration que opera na faixa 4.000 a $10.000 \mathrm{~cm}^{-1}$, com varredura de 16 scans e resolução $8 \mathrm{~cm}^{-1}$. Em cada face da amostra foram realizadas duas leituras, para obtenção de um espectro médio que representa a amostra (SILVA et al., 2012).

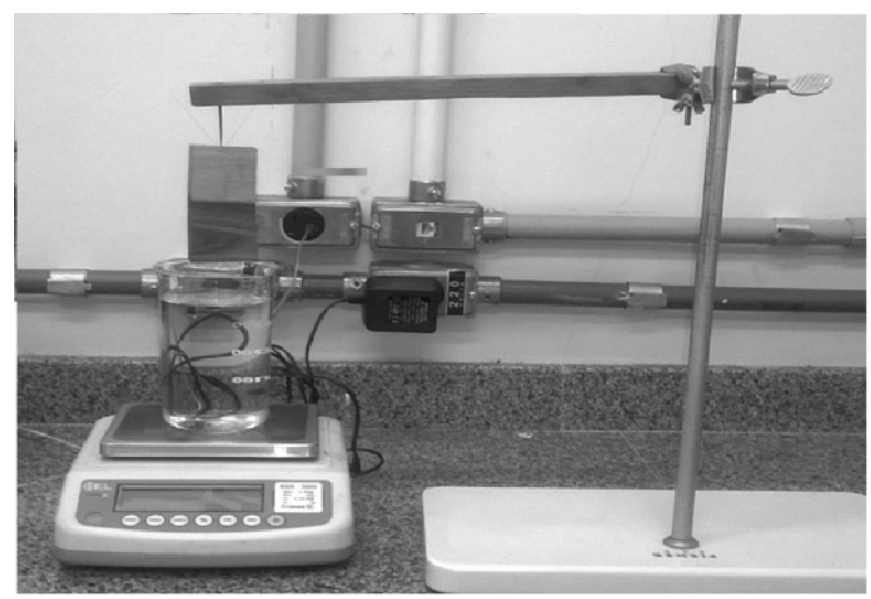

Figura 1. Instrumentação utilizada na determinação da densidade básica (Método convencional).

Figure 1. Instrumentation used to determine the basic density (Conventional method).

Modelos PLS (Regressão de mínimos quadrados parciais) foram desenvolvidos por Nascimento; Brasil (2016b) para predição da densidade básica, que se baseia na correlação dos espectros NIR (madeiras) com resultados dos ensaios pelo método tradicional da densidade básica, utilizando o software
TQ Analyst ${ }^{\mathrm{TM}}$. Assim, todos os resultados obtidos posteriormente para densidade, passam a ser não destrutivo, ou seja, o espectro NIR da madeira é obtido e correlacionado com os dados quimiométricos, uma similaridade do espectro novo é associada ao conjunto de dados.

O conjunto de dados (espectro NIR x valor da densidade) foi separado em dois grupos, o primeiro para calibrar a equação (2/3 dados) e um segundo para validar o modelo (1/3 dados). Foram testados três modelos preditivos, um que utiliza conjunto de dados brutos, e os outros dois com tratamento matemático $1^{\mathrm{a}} \mathrm{e} 2^{\mathrm{a}}$ derivação, com aplicação ou não dos filtros Norris e Savitzky-Golay. Para validação externa da espécie Eschweilera odora, foram utilizadas oito amostras para o plano radial e oito amostras para o plano tangencial.

A análise dos modelos preditivos foi baseada nos parâmetros estatísticos (quimiométricos), tais como: Maior coeficiente de regressão $\left(R^{2}\right)$ entre os valores de referência (metodologia destrutiva) e previstos (metodologia não destrutiva); menores erros de calibração (RMSEC - raiz quadrada do erro médio de calibração), previsão (RMSEP - raiz quadrada do erro médio de previsão); menor quantidade de variáveis latentes (VL) e a performance do modelo (real/predito da densidade básica) (ACUNA; MURPHY, 2006; NASCIMENTO; BRASIL, 2016b).

\section{Resultados e Discussão}

Os resultados da densidade básica das madeiras utilizadas na modelagem NIR (Tabela 2) variaram de média a alta $(0,55$ a $\left.0,94 \mathrm{~g} / \mathrm{cm}^{3}\right)$, representando a maioria das espécies arbóreas da Amazônia (INPA/CPPF, 1991; NAHUZ, 2013; SILVA et al., 2015).

Jati et al. (2014) estudando a densidade da madeira de árvores da região norte da Amazônia brasileira, identificou a predominância de madeira com densidade média (Curatella americana $=0,525 \mathrm{~g} / \mathrm{cm}^{3} ;$ Byrsonima crassifólia $=0,674$ 
$\left.\mathrm{g} / \mathrm{cm}^{3}\right)$. Madeiras proveniente da região do alto rio Negro/AM foram classificadas por Nascimento et al. (2012b) em média a alta densidade (Ocotea fragrantissima $=0,490 \mathrm{~g} / \mathrm{cm}^{3} ;$ Peltogyne catingae $=0,910 \mathrm{~g} / \mathrm{cm}^{3}$ ). Silveira et al. (2013) avaliando nove madeiras comerciais da Amazônia, também chegou ao patamar, média a alta densidade. Madeiras oriundas de plantio, como no caso de espécies de Pinus taeda e Eucalyptus sp., a classificação fica entre baixa a média densidade (MAGALHÃES et al., 2006; HEIN et al., 2010).

A densidade é uma propriedade importante da madeira que se relaciona estreitamente com a composição química e a estrutura anatômica (ZHU et al., 2009; VALE et al., 2010). Nuopponen et al. (2006) investigou a madeira de Picea sitchensis, Pinus silvestre e 24 espécies de madeiras tropicais, afim de estabelecer a relação entre a constituição química e a densidade da madeira, chegando à conclusão de que as madeiras tropicais obtiveram maior densidade que as madeiras de spruce e pinho, verificando ainda que as espécies tropicais apresentavam maior teor de lignina em sua composição, que as demais madeiras.
Vale et al. (2010) encontrou uma correlação positiva entre densidade básica versus teor de lignina, agrupando as espécies estudadas em duas categorias: Categoria formada com espécies de madeiras duras, densidade básica acima de $0,70 \mathrm{~g} / \mathrm{cm}^{3}$ e que apresentaram os maiores teores de lignina; A segunda foi formada por espécies com madeiras medianamente macias, e densidade $\leq 0,70 \mathrm{~g} / \mathrm{cm}^{3}$ e com os menores teores de lignina.

Castilho (1984) caracterizou a madeira de machimango colorado (Eschweilera iquitoensis) da Amazônia peruana, apresentando essa espécie cerca de 30\% de lignina, $8 \%$ de extrativos totais e 1\% de cinzas. Moutinho et al. (2011), encontrou valores aproximados para a lignina (27,6 a 30,8\%), quando estudou seis espécies Eschweilera da região de Paragominas/PA. Sendo que os teores de extrativos chegaram ao índice de 11,1\% para a espécie Eschweilera coriacea, e teor de cinzas de 2,9\% para a espécie Eschweilera grandiflora.

A modelagem PLS para densidade básica foi realizada nos dois planos (radial e tangencial), utilizando um universo amostral constituídos de 40 amostras para calibração, 24 amostras para validação interna, e ainda oito amostras para

Tabela 2. Valores da densidade básica das madeiras amazônicas determinadas pela metodologia tradicional.

Table 2. Values of the basic density of the Amazonian woods determined by the traditional methodology.

\begin{tabular}{|c|c|c|c|c|}
\hline \multirow{2}{*}{ Nome comercial/ Nome científico } & \multirow{2}{*}{$\begin{array}{l}\text { Número de } \\
\text { amostras }\end{array}$} & \multicolumn{3}{|c|}{ Densidade básica $\mathrm{g} / \mathrm{cm}^{3}$} \\
\hline & & Média* & Máximo & Mínimo \\
\hline Abiurana/ Manilkara amazoniaca & 04 & $0,92(0,0778)$ & 1,00 & 0,80 \\
\hline Abiurana/ Pouteria guianensis & 04 & $0,86(0,0707)$ & 0,93 & 0,79 \\
\hline Breu vermelho/ Protium tenuifolium & 08 & $0,76(0,0070)$ & 0,86 & 0,70 \\
\hline Breu vermelho/ Protium puncticulatum & 04 & $0,63(0,0212)$ & 0,66 & 0,60 \\
\hline Ingá vermelha/ Inga sp. & 04 & $0,94(0,0778)$ & 0,99 & 0,85 \\
\hline Ingá vermelha/ Inga paraenses & 04 & $0,66(0,0354)$ & 0,68 & 0,63 \\
\hline Ingá vermelha/ Inga alba & 04 & $0,55(0,0283)$ & 0,58 & 0,53 \\
\hline Muiragiboia/ Swartzia recurva & 04 & $0,80(0,0849)$ & 0,86 & 0,74 \\
\hline Murici/ Byrsonima crispa & 08 & $0,56(0,1414)$ & 0,67 & 0,45 \\
\hline Piãozinho/ Micrandopsis scleroxylon & 16 & $0,91(0,0495)$ & 0,99 & 0,78 \\
\hline Matá-matá/ Eschweilera sp. & 04 & $0,88(0,0707)$ & 0,93 & 0,80 \\
\hline
\end{tabular}

* valores em parênteses Desvio padrão 
validação externa. Na Figura 2 são apresentados os espectros NIR das madeiras da região amazônica, onde a análise espectral possibilitou associar os espectros $x$ densidade básica, indicando que amostras/espectro dispostos na região superior caracterizam espécies de maior densidade, e na região inferior as madeiras de média densidade.

Poletto et al. (2012) estudando as madeiras de Eucaliptus grandis, Dipterix odorata, Mezilaurus itauba e Pinus elliottii, observou que espécies com maior densidade apresentavam disposição do espectro infravermelho na região superior, tal como aconteceu com madeira de itaúba $\left(\mathrm{d}=0,70 \mathrm{~g} / \mathrm{cm}^{3}\right)$. Comportamento similar foi observado para madeiras de Eucalyptus globulus, Pinus radiata, Pinus palustris e Picea mariana (SCHIMLECK et al., 1999; SCHIMLECK et al., 2002; VIA et al., 2003; LEBLON et al., 2013). Esse comportamento pode estar associado à variação da absorção da madeira que está relacionada com seus componentes químicos, tais como a lignina e extrativos, e correlacionados com a densidade básica.

A faixa espectral utilizada na modelagem da densidade foi $4.000-10.000 \mathrm{~cm}^{-1}$, a utilização deste intervalo possibilita uma maior informação espectral correlacionada às propriedades químicas e físicas da madeira. A estatística multivariada permite avaliar o conjunto de dados, indicando uma maior similaridade em vários comprimentos do espectro. Estudos desenvolvidos para estimativa da densidade básica de Pinus maximinoi e Pinus tecunumanii (LAZZAROTTO et al., 2016), Eucaliptus benthammi, e Eucalipto pellita (LAZZAROTTO; MAGALHÃES, 2014) utilizaram o mesmo número de ondas desta pesquisa. Pigozzo (2009) testando a aplicabilidade da da ferramenta NIR para predizer a densidade básica de ma-

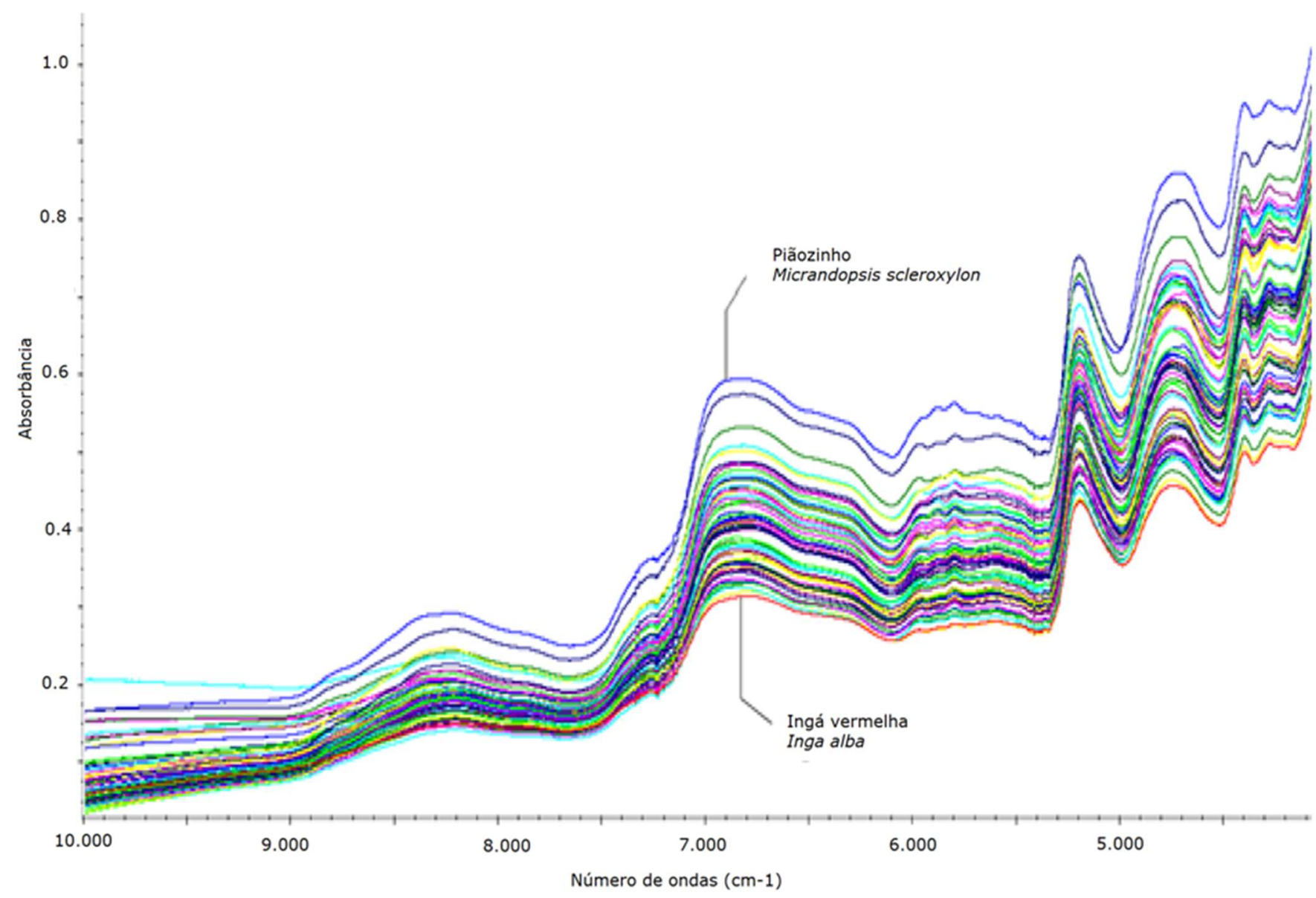

Figura 2. Universo espectral NIR com amostras de alta densidade e média densidade.

Figure 2. NIR spectral universe with high density and average density samples. 
Tabela 3. Modelos NIR para predição da densidade básica no plano radial (R) e tangencial (T).

Table 3. NIR models for prediction of basic density in the radial plane $(R)$ and the tangential plane $(T)$.

\begin{tabular}{|c|c|c|c|c|c|c|}
\hline Modelos & Tratamento/Filtro & $\mathbf{R}^{2}$ & RMSEC & RMSEP & VL & Performance \\
\hline $\mathrm{R} 1$ & Sem tratamento & 0,907 & 0,0714 & 0,0908 & 3 & 0,812 \\
\hline $\mathrm{R} 2$ & $1^{\text {a }}$ derivada Savitzky-Golay & 0,909 & 0,0704 & $\mathbf{0 , 0 7 3 7}$ & 2 & 1,032 \\
\hline $\mathrm{R} 3$ & $1^{\text {a }}$ derivada Norris & 0,906 & 0,0717 & 0,0761 & 2 & 0,968 \\
\hline $\mathrm{R} 4$ & $2^{\text {a derivada Savitzky-Golay }}$ & 0,999 & $0,00767 \mathrm{e}$ & 0,0831 & 7 & 0,876 \\
\hline R5 & $2^{\text {a }}$ derivada Norris & 0,907 & 0,0711 & 0,0777 & 2 & 1,080 \\
\hline $\mathrm{T} 1$ & Sem tratamento & 0,907 & 0,0711 & 0,1030 & 3 & 0,732 \\
\hline $\mathrm{T} 2$ & $1^{\text {a derivada Savitzky-Golay }}$ & 0,932 & 0,0615 & 0,0912 & 3 & 1,248 \\
\hline T3 & $1^{\text {a }}$ derivada Norris & 0,940 & 0,0576 & 0,0857 & 5 & 1,094 \\
\hline $\mathrm{T} 4$ & $2^{\text {a }}$ derivada Savitzky-Golay & 0,949 & 0,0531 & 0,0808 & 2 & 1,074 \\
\hline $\mathrm{T} 5$ & $2^{\text {a }}$ derivada Norris & 0,912 & 0,0695 & 0,0860 & 2 & 0,926 \\
\hline
\end{tabular}

$\mathrm{R}^{2}$ = coeficiente de regressão; RMSEC $=$ Raiz quadrada do erro médio de calibração; RMSEP $=$ Raiz quadrada do erro médio de predição; $\mathrm{VL}=$ variável latente.

deiras neotropicais, utilizou a faixa de $4.000-9.000 \mathrm{~cm}^{-1}$. Enquanto, Leblon et al. (2013) numa revisão do monitoramento da densidade da madeira pela espectroscopia do infravermelho próximo, verificou a utilização de $4.000-25.000 \mathrm{~cm}^{-1}$, para as madeiras de eucalipto, pinho, carvalho vermelho e larix.

Na Tabela 3 são apresentados o coeficiente de determinação $\left(\mathrm{R}^{2}\right)$ e erro padrão da calibração (RMSEC) e previsão (RMSEP) de cada modelo nos planos radial (R) e tangencial (T). Os modelos de predição da densidade básica nos dois planos ( $\mathrm{R} \mathrm{e} \mathrm{T}$ ) foram considerados satisfatórios visto que o coeficiente de regressão $>0,90$. O modelo $\mathrm{R}^{2}$ apresentou melhor desempenho estatístico com RMSEC $=0,0704 \mathrm{~g} / \mathrm{cm}^{3}, \mathrm{RMSEP}$ $=0,0737 \mathrm{~g} / \mathrm{cm}^{3} \mathrm{e} \mathrm{VL}=2$, enquanto o $\mathrm{T} 4$ obteve $\mathrm{RMSEC}=$ $0,0531 \mathrm{~g} / \mathrm{cm}^{3}, \mathrm{RMSEP}=0,0808 \mathrm{~g} / \mathrm{cm}^{3} \mathrm{e} \mathrm{VL}=2$. A performance dos modelos (real/predito da densidade básica) confirma a indicação dos modelos com melhor desempenho $(\sim 1,00)$.

Na Figura 3 são apresentados os gráficos da validação cruzada da densidade básica (calculada $x$ estimada) para as 64 amostras nos planos radial e tangencial. Como pode ser observado, as correlações em ambos os planos são altas $(>0,90)$ e apresentam baixos erros de calibração e predição.
A modelagem PLS (regressão de mínimos quadrados parciais) desenvolvida por Santos et al. (2009), para predição da densidade de Eucalyptus sp., resultou em um alto índice de correlação $(>0,90)$ e RMSEP de $0,077 \mathrm{~g} / \mathrm{cm}^{3}$, onde esse erro está próximo ao encontrado neste estudo para os modelos do plano radial (R2, R3 e R5). Enquanto, Via et al. (2003) utilizaram a regressão linear múltipla (MLR) para estimar a densidade básica da madeira Pinus, obteve na calibração de seus modelos $\mathrm{R}^{2} \leq 0,86$. O valor de $\mathrm{R}^{2}$ é uma medida da variável de resposta (densidade da madeira) que é explicada pelo modelo de regressão. Para materiais heterogêneos, tal como madeira, valores de $\mathrm{R}^{2}$ acima de 0,75 são considerados eficientes (KELLEY et al., 2004).

Na Figura 4 estão representados os valores da densidade básica determinada por método destrutivo (calculado) e estimado por meio das leituras dos espectros NIR nas faces tangenciais e radiais das amostras. $\mathrm{O}$ teste $\mathrm{T}$ mostrou que a diferença entre a predição PLS Radial e Tangencial (R2 e T4) não foi significativa, para um intervalo de confiança 95\%, ou seja, a predição da densidade da madeira no plano radial e tangen cial apresentou resultados aproximados. Este resultado con- 


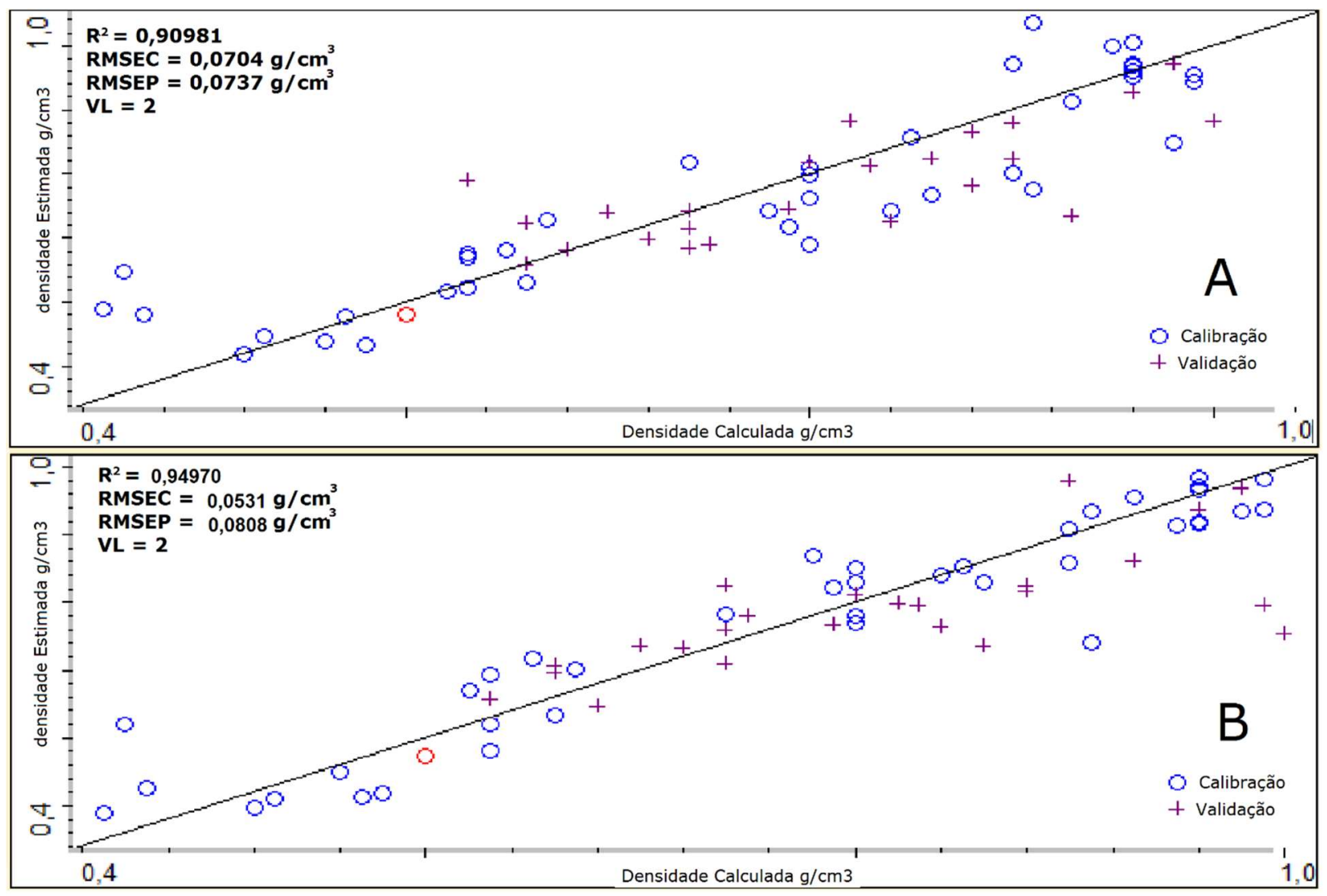

Figura 3. Curva de calibração e amostras validadas internamente com respectivos erros de calibração (RMSEC) e de previsão (RMSEP): A - plano radial; B - plano tangencial.

Figure 3. Calibration curve and internally validated samples with respective calibration errors (RMSEC) and prediction errors (RMSEP): A - radial plane; B - tangential plane.

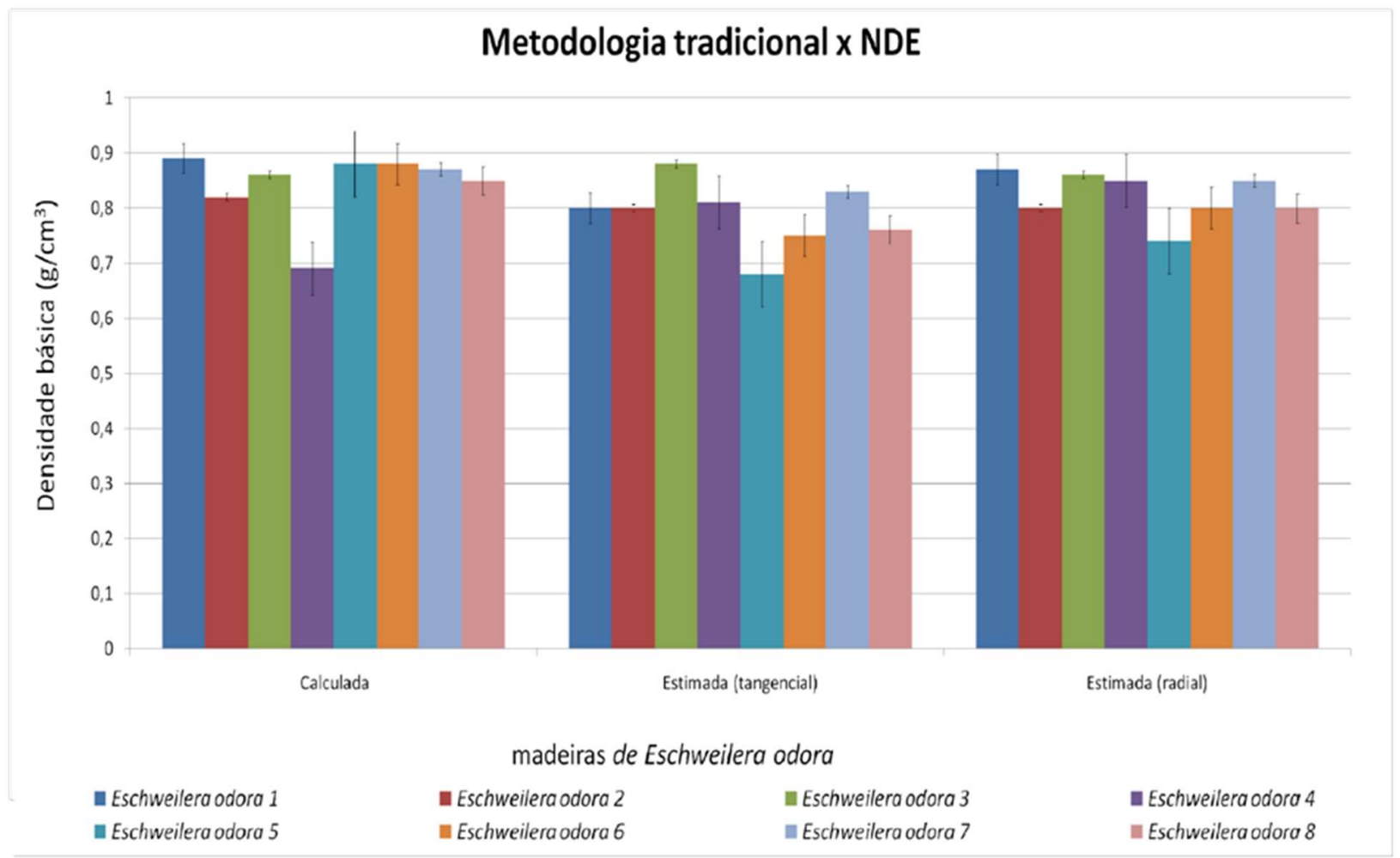

Figura 4. Validação dos valores da densidade básica da madeira de Eschweilera odora pela ferramenta NIR.

Figure 4. Validation of the values for the basic wood density of Eschweilera odora by the NIR tool. 
firma a potencialidade da metodologia NDE pela espectroscopia NIR, onde os valores estimados da densidade básica da madeira de matá-matá (Eschweilera odora) foram confirmados.

Gouveia et al. (2011) validando métodos não destrutivos para predição da densidade básica da madeira de eucalipto, verificou baixos erros de predição e uma precisão de $\leq 98 \%$ em comparação aos métodos tradicionais. Enquanto, Lazzarotto et al. (2016) validando a predição NIR de madeiras de pinho, encontrou uma boa precisão $(\leq 95 \%)$. O erro de predição da validação externa da madeira de Eschweilera odora ficou entre $0,03 \mathrm{~g} / \mathrm{cm}^{3}$ (radial) e $0,05 \mathrm{~g} / \mathrm{cm}^{3}$ (tangencial), evidenciando uma precisão 95\%, em comparação com a metodologia destrutiva.

\section{Conclusões}

A metodologia não destrutiva - NDE apresenta grandes vantagens quando aplicada à madeira e produtos acabados na caracterização tecnológica. A densidade da madeira de Eschweilera odora (matá-matá) foi validada na ferramenta NIR. Os modelos preditivos desenvolvidos a partir dos planos tangenciais e radiais forneceram estimativas de densidade semelhante, indicando que a aplicação da espectroscopia NIR em peças de madeiras tropicais pode ser realizada em qualquer um dos planos. A espectroscopia no infravermelho próximo (NIR) combinada com análise multivariada PLS apresenta resultados satisfatórios para predição da densidade básica de madeiras da Amazônia, potencializando esta ferramenta na área florestal.

\section{Referências}

ACUNA, M.A.; MURPHY, G.E. Use of near infrared spectroscopy and multivariate analysis to predict wood density of Douglas-fir from chain saw chips. Forest Products Journal, v.56 n.11/12, p. 67-72, 2006.
ASSOCIAÇÃO BRASILEIRA DE NORMAS TÉCNICAS (ABNT). NBR 7190: projeto de estruturas de madeira, Rio de Janeiro, 1997.

BIEKER, D.; RUST, S. Non-destructive estimation of sapwood and heartwood width in Scots pine (Pinus sylvestris L.). Silva fennica, v. 44 , n. 2 , p. $267-273,2010$

BODIG, J. The process of NDE research for wood and wood composites. In: $\mathbf{1 2}^{\text {th }}$ International symposium on nondestructicve testing of wood. Proceedings. University of Western Hungary, Sopron, 13-15 September, 2000.

CASTILLO, M.E.U. Determinacion del poder calorífico de 20 espécies florestales de la Amazonia peruana. Revista Florestal do Peru, v. 12, n. 1-2, p. 98-117, 1984.

GOUVÊA, A.F.G.; TRUGILHO, P.F.; GOMIDE, J.L.; DA SILVA, J.R.M.; ANDRADE, C.R.; ALVES, I.C.N. Determinação da densidade básica das madeiras de Eucalyptus por diferentes métodos não destrutivos. Árvore, v. 35, n. 2, p. 349358, 2011.

HAUKSSON, J. B.; BERGQVIST, G.; BERGSTEN, U.; SJOSTROM, M.; EDLUND, U. Prediction of basic wood properties for Norway spruce. Interpretation of Near Infrared Spectroscopy data using partial least squares regression. Wood Science and Technology, v.35, n.6, p. 475-481, 2001.

HEIN, P.R.G.; LIMA, J.T.; CHAIX, G. Otimização de calibrações baseadas em espectroscopia no infravermelho próximo para estimativa de propriedades da madeira de Eucalyptus. Floresta, v. 40, n. 3, p. 615-624, 2010.

INSTITUTO NACIONAL DE PESQUISAS DA AMAZÔNIA - INPA/CPPF. Catálogo de Madeiras da Amazônia. Área da Hidrelétrica de Balbina, INPA, Manaus/AM, 1991. $164 \mathrm{p}$.

JATI, S.R.; FEARNSIDE, P.M.; BARBOSA, R.I. Densidade da madeira de árvores em savanas do norte da Amazônia brasileira. Acta amazônica, v. 44, n. 1, p. 79-86, 2014.

JONES, P.D.; SCHIMLECK, L.R.; PETER, G.F.; DANIELS, R.F.; CLARK, A. Nondestructive estimation of Pinus taeda L. wood properties for samples from a wide range of sites in Georgia. Canadian Journal of Forest Research, v.35, p. 8592, 2005.

KELLEY, S.S.; RIALS, T.G.; SNELL, R.; GROOM, L.H.; SLUITER, A. Use of near infrared spectroscopy to measure 
the chemical and mechanical properties of solid wood. Wood Science and Technology, v.38, p. 257-276, 2004.

KRONGTAEW, C.; MESSNER, K.; TRES, T.; FACKLER, K. Characterization of key parameters for biotechnological lignocelluloses conversion assessed by FT-NIR spectroscopy Part I: Qualitative analysis of pretreated straw. BioResources. v. 5, n. 4, p. 2063-2080, 2010.

LAZZAROTTO, M.; NETIPANYJ, R.R, MAGALHÃES, W.L.E; DE AGUIAR, A.V. Espectroscopia no infravermelho próximo para estimativa da densidade básica de madeiras de Pinus. Brazilian Journal of Wood Science, v. 7, n. 3, p. 119126, 2016.

LAZZAROTTO, M.; MAGALHÃES, W.L.E. Uso da espectroscopia no infravermelho próximo (NIR) para predição não-destrutiva de densidade básica da madeira de Eucalyptus benthammi e Eucalyptus pellita. Comunicado Técnico - Embrapa Florestas, n. 334, p. 1-6, 2014.

LEBLON, B.; ADEDIPE, O.; HANS, G.; HADDADI, A.; TSUCHIKAWA, S.; BURGER, J.; STIRLING, R.; PIROUZ, Z.; GROVES, K.; NADER, J.; LAROCQUE, A. A review of near-infrared spectroscopy for monitoring moisture content and density of solid wood. The Forestry Chronicle, vol. 89, n. 5. p. 595-606, 2013.

LIRA, J.N.; NASCIMENTO, C.C.; PAULA, E.V.M. Avaliação da densidade básica da madeira do cerne e alburno de oito espécies de madeiras da Amazônia. In: 62 a Reunião SBPC, 2010, Natal. Anais da 62a Reunião Anual da SBPC, 2010. v. 1.p. $1-1$.

MAGALHÃES, W.L.E.; ZANGISKI, F.; Klein, C.H.; Higa, A.R.; Uso da espectroscopia no infravermelho próximo (NIR) para predição não-destrutiva de densidade básica da madeira de Pinus taeda. Comunicado Técnico - Embrapa Florestas, n. 159, p. 1-4, 2006.

MOUTINHO, V.H.P.; COUTO, A.M.; LIMA, J.T.; DE AGUIAR, O.J.R.; NOGUEIRA, M.O.G. Caracterização Energética de madeiras de espécies conhecidas na Amazônia brasileira como Matá-Matá (Eschweilera Mart Ex Dc). Scientia Forestalis. For., v. 39, n. 92, p. 457-461, 2011.

MUÑIZ, G.I.B.; CARNEIRO, M.E.; NISGOSKI, S.; RAMIREZ, M.G.L.; MAGALHÃES, W.L.E. SEM and NIR characterization of four charcoal species. Wood Science and Technology, v. 47, n.4, p. 815-823, 2013.
GRACIELA INÉS BOLZON DE MUÑIZ, G.I.B.; CARNEIRO, M.E.; BATISTA, F.R.R., SCHARDOSIN, F.Z.; NISGOSKI, S. Wood and charcoal identification of five species from the miscellaneous group known in Brazil as "angelim" by Near-IR and wood anatomy. Maderas. ciencia y tecnología, v. 18, n. 3, p. 505 - 522, 2016.

NAHUZ, A.R. Catálogo de madeiras brasileiras para a construção civil. São Paulo: IPT, 2013, 103 p.

NASCIMENTO, C. C. Variabilidade e desenvolvimento de modelos para estimar propriedades mecânicas da madeira. Manaus: INPA/ FUA, 2000. 120 p.

NASCIMENTO, C.C.; LIMA, M.P.; BRASIL, M.M.; ARAUJO, R.D.; PAULA, E.V.C.M. Desenvolvimento de (Bio) tecnologias para aproveitamento de recursos naturais no alto rio negro. In: Souza, L.A.G; Guillermo-Castellón, E. (Org.). Desvendado as Fronteiras do conhecimento na região Amazônica do alto rio Negro. Manaus: INPA - Projeto FRONTEIRAS, p. 69-84. 2012a.

NASCIMENTO, C.S.; VAREJÃO, M.J.C.; VIANEZ, B.F. Espectroscopia de infravermelho próximo com transformada de Fourier na predição de extrativos e polifenóis totais em cascas de espécies florestais da Amazônia. In: Vianez et al. (Org.). Potencial tecnológico de madeiras e resíduos florestais da Amazônia Central. Manaus: INPA, p. 213-224, 2012b.

NASCIMENTO. C.C; BRASIL, M.M. Uso da espectroscopia de infravermelho próximo como ferramenta para classificação da madeira. In: IV SICASA e I ANPPAS Amazônia. Anais. Universidade Federal do Amazonas, Manaus, 19-22 Setembro, 2016a.

NASCIMENTO. C.C; BRASIL, M.M. Modelo NIR para predição da densidade básica de madeiras da Amazônia (software). Manaus - AM, Brasil. LEAM/INPA, 2016 b.

NIEMZA, P.; MANNES, D. Non-destructive testing of wood and wood-based materials. Journal of Cultural Heritage, v. 13, p. 26-34, 2012.

NUOPPONEN, M.H.; BIRCH, G.M; SYKES, R.J.; LEE, S.J.; STEWART, D. Estimation of wood density and chemical composition by means of diffuse reflectance mid-infrared Fourier transform (DRIFT-MIR) spectroscopy. Journal Agriculture Food Chemical, v. 4, n.1, p. 34-40, 2006. 
OLIVEIRA, F.G.R.; CAMPOS, J.A.O.; PLETZ, E.; SALES. A Non destructive evaluation of Wood using ultrasonic technique. Madera, ciencia y tecnologia, v.4, n.2, p. 133-139, 2002.

PIGOZZO, R.J.B. Aplicabilidade do infravermelho-próximo (NIR) na identificação e estimativa de propriedades da madeira. Madeira, arquitetura e engenharia. v. 10, n. 25, p. 59$61,2009$.

POLETTO, M.; ZATTERA, A.J; SANTANA, R.M.C. Structural differences between wood species: evidence from chemical composition, FTIR spectroscopy and thermogravimetric analysis. Journal of Applied Polymer Science, v. 126, p. 337 $344,2012$.

ROSS, R.J.; WANG, X.; MATTSON, J.A.; ERICKSON, J.R.; FORSMASN, J.W.; GESKE.E.A.; WEHR, M.A. Comparison of several nondestructive evaluation technique for assessing stiffness and MOR of small diameter logs. In: $12^{\text {th }}$ International Symposium on Nondestructicve Testing of Wood. Proceedings. University of Western Hungary, Sopron, 13-15 September, 2000.

SANABRIA, A.S.; FURRER, R.; NEUENSCHWANDER, J.; NIEMZ, P.; SENNHAUSER, U. Aircoupled ultrasound inspection of glued laminated timber, Holzforschung, v. 65, p. 377-387, 2011.

SANTOS, A.J.A.; ANJOS, O.; PEREIRA, H. Estimation of Acacia melanoxylon unbleached Kraft pulp brightness by NIR spectroscopy. Forest Systems. v. 24, n. 2, p. 1-6, 2015.

SANTOS, R.B.; GOMIDES, J.L.; SOUSA, L.C. Predição de qualidade da madeira e da polpa celulósica por técnica de espectroscopia de infravermelho próximo (NIRS). Árvore, v. 33, n. 4, p. 759-767, 2009.

SCHIMLECK, L.R.; EVANS, R.; MATHESON, A.C. Estimation of Pinus radiate D. Don clear wood properties by near Infrared spectroscopy. Journal of Wood Science, v. 48, p. 132-137, 2002.

SCHIMLECK, L. R. et al. Estimation of basic density of Eucalyptus globulus using near-infrared spectroscopy. Canadian Journal of Forest Research, v.29, n.2, p.194-201, 1999.

SCHIMLECK, L. R.; EVANS, R.; ILIC, J. Estimation of Eucalyptus delegatensis wood properties by Near Infrared Spectroscopy. Canadian Journal of Forest Research, v.31, p. 1671-1675, 2001.
SILVA, C.J.; VALE, A.T.; MIGUEL, E.P. Densidade básica da madeira de espécies de cerradão no estado de Tocantins. Pesquisa Florestal Brasileira. v. 35, n. 82, p. 63-75, 2015.

SILVA, C.E.; HIGUCHI, N.; NASCIMENTO, C.C.; DURGANTE, F.; BRASIL, M.M. Use of near Infrared Spectroscopy (FT-NIR) to predict wood density of three Brosimum species (Moraceae) of alto Rio Solimões. In: 2012 IAWA Pan Americano Meeting, Proceedings. International Association of Wood Anatomists - IAWA, Recife, 1-5 October, 2012.

SILVA, C.E.; HIGUCHI, N.; NASCIMENTO, C.C.; DURGANTE, F.; BRASIL, M.M. Bioprospecção da densidade básica por FT-NIR de cinco espécies de madeira da família Lauraceae do Oeste da Amazônia. In: I Congresso Brasileiro de Tecnologia da Madeira. Anais. UFRRJ, Petrópolis, 2013. p.430-431.

SOUSA, L.C.; GOMIDE, J.L.; MILAGRES, F.R.; ALMEIDA, D.P. Desenvolvimento de modelos de calibração NIRS para minimização das análises de madeira de Eucalyptus spp. Ciência Florestal. v. 21, n. 3, p. 591-599, 2011.

STANGERLIN, D.M.; CORASSA, J.N.; GATTO, D.A.; PEREIRA, R.L.; CASTELO, P.A.R. Caracterização mecânica de madeiras deterioradas em campo por meio de ultrassom e flexão estática. Comunicata Scientiae, v.6, n.3, p. 365-372, 2015.

TANASOIU, V.; MICLEA, C.; TANASOIU, C. Nondestructive testing thechniques and piezoeletric ultrasonics transducers for wood and buil in wooden structures. Journal of Optoeletrics and Advanced Materials, v. 4, n.4, p. 949-957, 2002.

TERDWONGWORAKUL, A.; PUNSUWAN, V.; THANAPASE, W.; TSUCHIKAWA, S. Rapid assessment of wood chemical properties and pulp yield of Eucalyptus camaldulensis in Thailand tree plantations by near infrared spectroscopy for improving wood selection for high quality pulp. Journal Wood Science, v.51, n.2, p. 167-171, 2005.

TSUCHIKAWA, S. A review of recent near infrared research for wood and paper. Applied Spectroscopy Reviews, v.42, p. 43-71, 2007.

TSUCHIKAWA, S.; INOUE, K.; NOMA, J.; HAYASHI, K. Application of near-infrared spectroscopy to wood discrimination. Journal Wood Science, v.49, p. 29-35, 2003.

VALE, A.T.; DIAS, I.S.; SANTANA, M.A.E. Relações entre propriedades químicas, físicas e energéticas da madeira de 
cinco espécies de Cerrado. Ciência Florestal, v. 20, n. 1, p. 137-145, 2010.

VAREJÃO, M. J. C.; NASCIMENTO, C. S.; CRUZ, I. A. Avançando fronteiras: potencial químico, ecológico-econômico de espécies florestais de São Gabriel da Cachoeira, AM. In: Souza, L.A.G; Guillermo-Castellón, E. (Org.). Desvendado as Fronteiras do conhecimento na região Amazônica do alto rio Negro. Manaus: INPA - Projeto FRONTEIRAS, p. 51-67, 2012.

VIA, B.K.; SHUPE, T.F.; GROOM, L.H.; STINE, M. SO, C-H. Multivariate modelling of density, strength and stiffness from near infrared spectra for mature, juvenile and pith wood of longleaf pine (Pinus palustris). Journal of Near Infrared Spectroscopy, v.11, p. 365-378, 2003.

ZAUNER, M.; KEUNECKE, D.; STAMPANONI, M.; NIEMZ, P. Synchrotron based tomographic microscopy of wood under compression, Bulletin International Academia Wood Science. v. 2011-I, p. 25-27, 2011.

XIANGRONG ZHUA, X.; SHANA, Y.; LI, G.; HUANGC, A.; ZHANGD, Z. Prediction of wood property in Chinese Fir based on visible/near-infrared spectroscopy and least squaresupport vector machine. Spectrochimica Acta, v. 74, p. 344 $348,2009$. 